\title{
BMJ Open Comparative efficacy of once-weekly semaglutide versus SGLT-2 inhibitors in patients inadequately controlled with one to two oral antidiabetic drugs: a systematic literature review and network meta-analysis
}

\author{
Steve Kanters, ${ }^{1}$ Lars Wilkinson, ${ }^{2}$ Hrvoje Vrazic, ${ }^{2}$ Rohini Sharma, ${ }^{1}$ Sandra Lopes, ${ }^{2}$ \\ Evan Popoff, ${ }^{1}$ Eric Druyts ${ }^{1}$
}

To cite: Kanters S, Wilkinson L, Vrazic $\mathrm{H}$, et al. Comparative efficacy of once-weekly semaglutide versus SGLT2 inhibitors in patients inadequately controlled with one to two oral antidiabetic drugs: a systematic literature review and network meta-analysis. BMJ Open 2019;9:e023458. doi:10.1136/ bmjopen-2018-023458

- Prepublication history and additional material for this paper are available online. To view these files, please visit the journal online (http://dx.doi. org/10.1136/bmjopen-2018023458).

Received 9 April 2018

Revised 9 April 2019

Accepted 13 June 2019
Check for updates

(C) Author(s) (or their employer(s)) 2019. Re-use permitted under CC BY-NC. No commercial re-use. See rights and permissions. Published by BMJ.

${ }^{1}$ Precision Xtract, Vancouver, British Columbia, Canada ${ }^{2}$ Novo Nordisk A/S, Søborg, Denmark

Correspondence to

Steve Kanters;

skanterz@gmail.com

\section{ABSTRACT}

Objective To determine the comparative efficacy of once-weekly semaglutide relative to sodium-glucose cotransporter 2 inhibitors (SGLT-2is) licensed in Europe and North America among patients with type 2 diabetes (T2D) inadequately controlled with 1-2 oral antidiabetics (OADs), using a network meta-analysis (NMA). Design systematic review and network meta-analysis. Data Sources EMBASE, MEDLINE and CENTRAL were searched from January 1994 to August 2017.

Methods Randomised controlled trials with $\geq 20$ weeks of treatment evaluating once-weekly semaglutide or SGLT-2is. Primary outcomes included change from baseline in: HbA1c, weight, systolic blood pressure, postprandial blood glucose and fasting plasma glucose. Fixed-effect and random-effect Bayesian NMA were used to indirectly compare treatment effects at $26( \pm 4)$ weeks. Metaregression and sensitivity analyses were conducted. Model selection was performed using the deviance information criterion and consistency was assessed by comparing indirect (edge-splitting) to direct evidence.

Results Forty-eight publications representing 21 trials were included. The mean differences (MD) in change from baseline in $\mathrm{HbA} 1 \mathrm{c}$ of once-weekly semaglutide $1.0 \mathrm{mg}$ versus SGLT-2is ranged from $-0.56 \%$ for canagliflozin $300 \mathrm{mg}$ (95\% credible interval (Crl): -0.76 to $-0.33 \%)$, to $-0.95 \%$ for dapagliflozin $5 \mathrm{mg}$ ( $95 \% \mathrm{Crl}:-1.20$ to $-0.69 \%)$. The MD in change from baseline in weight of once-weekly semaglutide $1.0 \mathrm{mg}$ versus SGLT-2is ranged from $-1.35 \mathrm{~kg}$ for canagliflozin $300 \mathrm{mg}$ to -2.48 $\mathrm{kg}$ for dapagliflozin $5 \mathrm{mg}$, while change from baseline in fasting plasma glucose ranged from $-0.41 \mathrm{mmol} / \mathrm{L}$ for canagliflozin $300 \mathrm{mg}$ to $-1.37 \mathrm{mmol} / \mathrm{L}$ for dapagliflozin $5 \mathrm{mg}$. Once-weekly semaglutide was not statistically differentiable than all SGLT-2is in reducing systolic blood pressure. NMA was not feasible for postprandial blood glucose and safety outcomes.

Conclusion Once-weekly semaglutide demonstrated statistically significant and clinically meaningful reductions in $\mathrm{HbA} 1 \mathrm{c}$ and body weight in T2D patients inadequately
Strengths and limitations of this study

- Use of highly sensitive searches in the peer-reviewed literature supplemented with hand searches of leading diabetes research conferences.

- High quality of study data from the involvement of two independent researchers for study selection and data extraction.

- Robust analytical results from a variety of sensitivity analyses conducted.

- Limited patient and study characteristics from conference proceedings leading to difficulties in determining population heterogeneity.

- Lack of direct comparisons from the shortage of reported comparisons in available literature.

controlled with 1-2 OADs compared to all SGLT-2is licensed in Europe and North America.

\section{INTRODUCTION}

Type 2 diabetes (T2D) is a chronic condition characterised by a reduction in both insulin production and pancreatic beta-cell function, as well as by increased insulin resistance. ${ }^{1}$ In 2017, it was estimated that 425 million adults were living with diabetes, with approximately $90 \%$ of cases being T2D. ${ }^{2}$ Over the past decades, the prevalence of diabetes has been steadily rising. In 2017 , there were up to 4 million deaths directly caused by, or related to, diabetes. ${ }^{2}$ Effective treatments to help improve glycaemic control are essential to curbing the high morbidity and mortality of this global diabetes epidemic.

According to the American Diabetes Association (ADA), European Association for the Study of Diabetes (EASD) and National 
Institute for Clinical Excellence (NICE) UK guidance on T2D, metformin, an oral antidiabetic drug (OAD), should be considered as first-line treatment. ${ }^{3-5}$ The first stage of intensification following treatment failure consists of either adding another antidiabetic agent, such as dipeptidyl peptodase-4 inhibitor, pioglitazone or sulfonylurea, to metformin. ${ }^{6}$ Following failure of these treatments, a triple therapy is considered. Some guidelines, such ADA, ${ }^{7}$ include glucagon-like peptide-1 (GLP-1) receptor agonist and insulin-based therapies as considerations for both dual and triple therapy, while others, such as NICE, ${ }^{6}$ include them only as considerations for triple therapy.

Once-weekly semaglutide belongs to the GLP-1 receptor agonist drug class. It has shown promising results in the Semaglutide Unabated Sustainability in Treatment of T2D (SUSTAIN) programme, a series of global phase III randomised controlled trials (RCTs) of once-weekly semaglutide encompassing more than 8000 people with T2D. Sodium-glucose cotransporter 2 inhibitors (SGLT-2is) are another class of antidiabetics that have been approved by regulatory agencies within the past decade. Evidence has shown that these are potential options for a variety of treatment-experienced T2D populations.

At present, there is no direct evidence comparing the efficacy of once-weekly semaglutide relative to SGLT-2is. Despite the lack of head-to-head evidence, network meta-analyses (NMA) can be used to make indirect comparisons and to simultaneously review the therapeutic landscape. ${ }^{8-11}$ These methods use the evidence base of RCTs comparing the interventions to each other and select common comparators, as identified through systematic literature reviews (SLRs).

We sought to conduct an SLR and NMA to determine the efficacy of once-weekly semaglutide relative to SGLT-2is licensed in both Europe and North America among patients (aged $\geq 18$ years) with T2D with inadequate glycaemic control using 1-2 OADs. A separate study has evaluated the comparative efficacy and safety of once-weekly semaglutide and SGLT-2i in T2D patients inadequately controlled with metformin. ${ }^{12}$

\section{METHODS}

\section{Systematic literature review}

An SLR to identify pertinent RCTs was performed by searching MEDLINE, EMBASE and CENTRAL through Ovid from January 1994 to 5 April 2016, with updates on 3 October 2016 and 16 August 2017. Manual searches of the proceedings from four conferences published 20132017 were also performed: International Society for Pharmacoeconomics and Outcomes Research, International Diabetes Federation, EASD and ADA scientific sessions. The search strategy used in this study was designed for a larger SLR with highly sensitive search algorithms that ran concurrently and allowed for most currently used diabetes pharmacotherapies and for a more diverse population. The search strategies were shared with the sister study, but study selection and subsequent steps were separate. $^{12}$

All steps of the SLR, including study selection, were conducted in dual and independently. Discrepancies were resolved through consensus and a third reviewer was used as needed. Study eligibility criteria were defined a priori according to the population, interventions, comparisons, outcomes and study design (PICOS) to ensure that potentially relevant studies were selected in a systematic manner with a minimal risk of introducing bias in accordance with guidance published by the Centre for Reviews and Dissemination, and the Cochrane Handbook for Systematic Reviews. ${ }^{13}{ }^{14}$ Studies published in English meeting the following criteria were included; (1) adults aged 18 years or older with T2D inadequately controlled with 1-2 prior OADs; (2) 1.0 or $0.5 \mathrm{mg}$ doses of once-weekly semaglutide, or any approved doses of SGLT-2is that are licensed in Europe and North America; (3) reported one or more efficacy outcome(s); change from baseline $\mathrm{Hb}_{\mathrm{Alc}}$, weight, body mass index (BMI), and systolic blood pressure (SBP), postprandial blood glucose (PPG), fasting plasma glucose (FPG), proportion of patients achieving $<7 \%$ or $\leq 6.5 \%$ $\mathrm{Hb}_{\mathrm{Alc}}$, proportion of patients achieving $\geq 5$ or $10 \%$ wt loss, safety outcomes and triple composite outcome (based on reaching $<7.0 \% \mathrm{HbA}_{1 \mathrm{c}}$, having no hypoglycaemic events and no weight gain) and (4) RCT studies with treatment duration of as low as 20 weeks, including cross-over trials with a minimum 20-week assessment of efficacy outcomes at cross-over, because this was viewed as the amount of time for an antidiabetic to have its full effect on HbAlc, the primary outcome to most clinical studies of interest. ${ }^{15}$ In addition, only phase III trials were included in order to align the results with the SUSTAIN clinical trial study design. See online supplementary appendix for the full PICOS statement.

Data were extracted in duplicate using a standardised tool from the final list of eligible studies. Change from baseline data was extracted for continuous outcomes. In cases where change from baseline was not available, appropriate information to calculate change from baseline was extracted (see online supplementary appendix). Proportion of patients in each treatment arm with the event data was extracted for dichotomous outcomes. In addition, information on study design characteristics, inclusion/exclusion criteria, patient characteristics, interventions, definition and results for the outcomes of interest and other contextual factors was extracted.

The quality of individual trials was assessed across six conceptual domains using the Cochrane Risk of Bias Assessment ${ }^{14}$; selection bias, performance bias, detection bias, attrition bias, reporting bias and other sources of bias. Each domain was scored as low, unclear or high risk of bias, with disagreements again resolved by consensus discussions with a third reviewer.

\section{Analysis methods}

In order to gauge the suitability of conducting an NMA, a feasibility assessment was conducted to assess: 
(1) whether the RCT evidence for the interventions of interest formed a connected evidence network for each outcome of interest and time point; (2) the homogeneity of outcomes reported and data time points and (3) the distribution of study characteristics, subject characteristics and disease definitions that may impact treatment effects across direct comparisons of the evidence network for outcomes and time points of interest. ${ }^{1016}$

Based on the findings of the feasibility assessment, the results of the RCTs that formed a complete evidence network, and were deemed comparable, were synthesised by means of NMA to determine the relative treatment efficacy of once-weekly semaglutide, canagliflozin, dapagliflozin and empagliflozin at 26-week time points ( \pm 4 weeks), across the outcomes of interest.

Analyses were performed within a Bayesian framework, which involves data, a likelihood distribution, a model with parameters and prior distributions. The model relates the data from the individual studies to basic parameters reflecting the (pooled) relative treatment effect of each intervention compared with an overall reference treatment. Based on these basic parameters, the relative efficacy between each of the competing interventions was obtained. All analyses were conducted using non-informative priors in line with NICE technical support documents (see model code in online supplementary appendix). ${ }^{8}$

Fixed-effect or random-effect models were applied depending on values for the deviance information criterion (DIC). A difference in DIC of about three points was considered meaningful and the model with lowest DIC was selected as best-fit model. ${ }^{17}$ Where binary data were analysed, a logit link was used in the standard normal NMA regression setup to obtain ORs and relative risks (RRs) for each comparison in the network. For continuous outcomes, a normal likelihood NMA setup with an identity link function was used to calculate mean difference (MD) as the relative treatment effect.

For the NMA, ranking probabilities were estimated for all treatments of being at each rank for each intervention. A treatment hierarchy was determined using the probability of being the best treatment by using the surface under the cumulative ranking curve (SUCRA; score of $0-1$ where 1 is best). To determine whether there were issues of inconsistency, independent means models, to evaluate direct evidence, and edge-splitting, to evaluate indirect evidence, were used to generate two posterior distributions for the mean treatment effect. ${ }^{18}$ The difference in posterior means generated by the two sets of evidences was then used as an estimate of inconsistency within the network.

In our feasibility assessment, we found that NMA was not possible for the following outcomes: proportion of patients achieving $\leq 6.5 \% \mathrm{HbAlc}$, proportion of patients achieving $\geq 5$ or $10 \%$ wt loss, BMI, PPG ( $\mathrm{mmol} / \mathrm{L})$, safety outcomes and triple composite outcome. This was either because the network was disconnected between onceweekly semaglutide and SGLT-2is or the data were not available at $26 \pm 4$ weeks.
In addition to the principal analyses, the following sensitivity analyses were conducted: (i) removal of three trials with more than $40 \%$ Asian patients, ${ }^{19-21}$ (this is due to Asian ethnicity being a well-recognised effect modifier in diabetic therapeutics due to a greater level of B-cell dysfunction in East Asians) ${ }^{22-24}$; and (ii) removal of one trial with high cardiovascular risk. ${ }^{25}$ In our feasibility assessment, we observed differences between different direct comparisons in the distribution of the following characteristics: sex, disease duration, weight at baseline, $\mathrm{HbA}_{1 \mathrm{c}}$ at baseline, number of OADs failed at baseline. As such, we conducted metaregression analyses using these variables.

\section{Patient and public involvement}

Patients and public were not involved in the development of this study as it made use of publicly available data.

\section{RESULTS}

Figure 1 displays Preferred Reporting Items for Systematic Reviews and Meta-Analysesflow diagram, showing results separately for the original search and the two updates. A total of 38828 citations were identified from the three searches including 38814 via MEDLINE, EMBASE and CENTRAL, and 14 records identified through other sources. Studies in T2D patients with inadequate control on 1-2 OADs were then selected from this large evidence base. The most common reasons for study exclusion were study design, followed by an intervention or population outside the PICOS eligibility criteria. This resulted in 48 publications representing 21 trials included in the SLR.

The complete network of evidence is shown in figure 2 and networks by clinical outcome can be found in online supplementary appendix. Studies were similar with respect to trial characteristics (table 1) and baseline patient characteristics (table 2 and table 3 ). No evidence of inconsistency was observed in any of the outcomes. Tables on model selection and figures comparing the agreement of direct and indirect evidences are shown in the online supplementary appendix.

A majority of the trials were multicentre, phase III, double-blind trials. The only open-label trials were SUSTAIN $3^{26}$ and SUSTAIN $7 .^{27}$ Duration of follow-up was reported in all 21 trials, ranging from 24 to 104 weeks; mean age ranged from 53.5 to 61.0 years ${ }^{25}{ }^{28}$; mean weight at baseline ranged from 76.9 to $96.0 \mathrm{~kg}^{26} 29$; mean SBP ranged from 126 to $135 \mathrm{~mm} \mathrm{Hg}^{2130}$ and mean baseline HbA1c ranged from $7.2 \%$ to $9.3 \% .{ }^{31}{ }^{32}$ To note, all trials reported results at the 26-week time point, with the exception of SUSTAIN $2{ }^{33}$ SUSTAIN $3^{26}$ SUSTAIN $4{ }^{34}$ (23-week time point) and SUSTAIN 7 (28-week time point). ${ }^{27}$

The trials were considered to have low-risk of bias based on the assessment using Cochrane Risk of Bias Assessment tool. ${ }^{14}$ The only source of high-risk bias came from the lack of blinding and selective reporting in few included trials. Full trial and patient characteristics, and risk of bias 


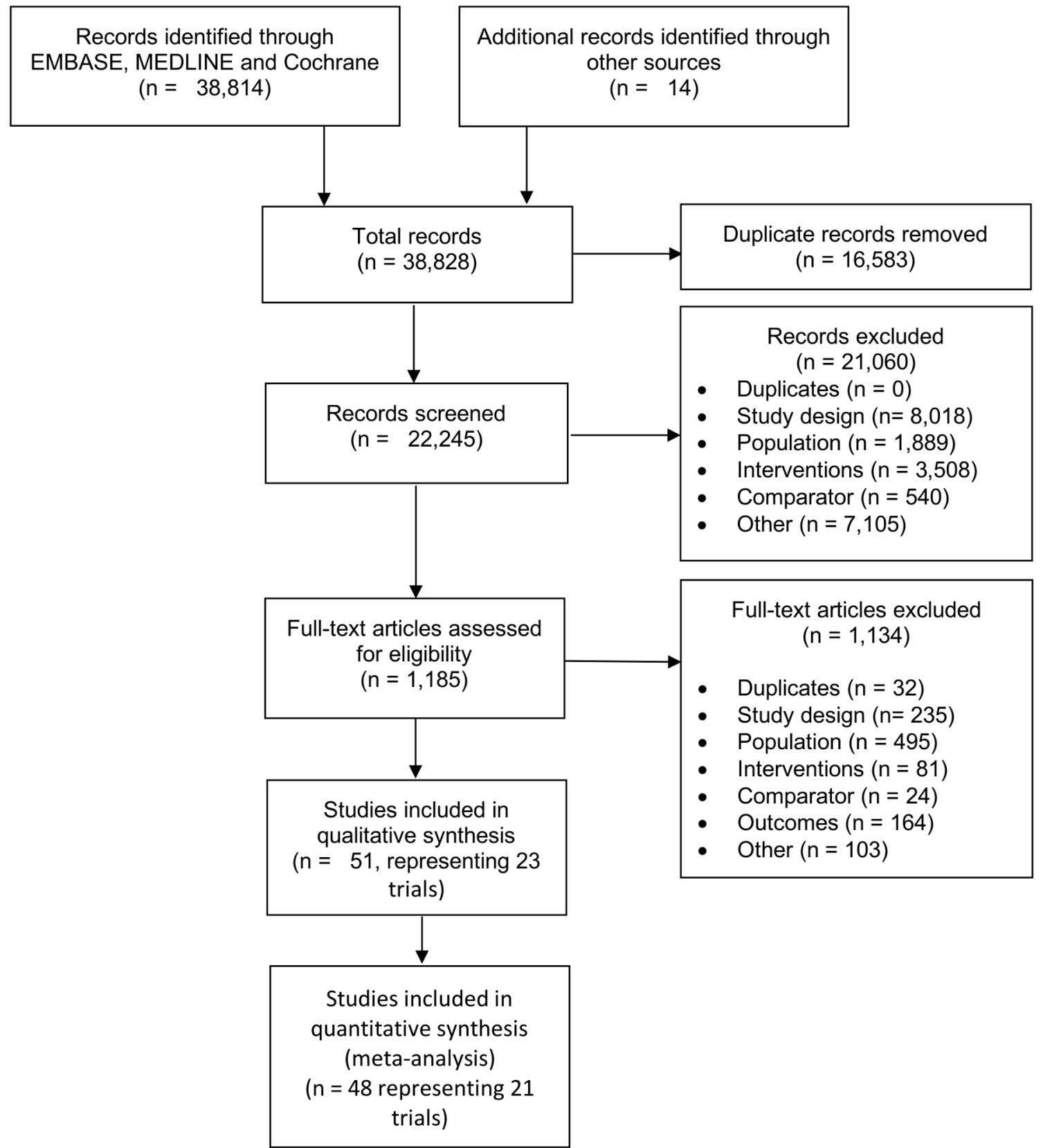

Figure 1 Preferred Reporting Items for Systematic Reviews and Meta-Analyses flow diagram, showing results separately for the original search and the two updates. A total of 38828 citations were identified from the three searches including 38814 via MEDLINE, EMBASE and CENTRAL, and 14 records identified through other sources. Studies in T2D patients with inadequate control on 1-2 OADs were then selected from this large evidence base. The most common reasons for study exclusion were study design, followed by an intervention or population outside the PICOS eligibility criteria. This resulted in 48 publications representing 21 trials included in the SLR. OADs, oral antidiabetics; SLRs, systematic literature reviews; T2D, type 2 diabetes

assessments, are provided in the online supplementary appendix.

\section{Change in $\mathrm{HbA}_{1 \mathrm{c}}$}

Change from baseline in $\mathrm{HbA}_{1 \mathrm{c}}$ was the primary outcome for most of the included trials. Twenty-one trials including 53 treatment arms and 11714 patients reported on 11 treatments. ${ }^{19-21} 25-28$ 32-45 The random-effect model was chosen as best-fit model because it had the lowest DIC (94.37).
As shown in table 4, there was a strong evidence that once-weekly semaglutide was more efficacious at reducing $\mathrm{HbA}_{1 \mathrm{c}}$ relative to SGLT-2is, at both doses. The $\mathrm{MD}$ in change from baseline in $\mathrm{HbA}_{1 \mathrm{c}}$ of once-weekly semaglutide $1.0 \mathrm{mg}$ versus SGLT-2is ranged from $-0.56 \%$ for canagliflozin $300 \mathrm{mg}$ (95\% credible interval (CrI): -0.76 to $0.33 \%)$ to $-0.95 \%$ for dapagliflozin $5 \mathrm{mg}(95 \%$ CrI: -1.20 to $-0.69 \%$ ) indicating that once-weekly semaglutide $1.0 \mathrm{mg}$ was statistically significantly more effective at reducing $\mathrm{HbA}_{1 \mathrm{c}}$. Statistically significant reductions in 


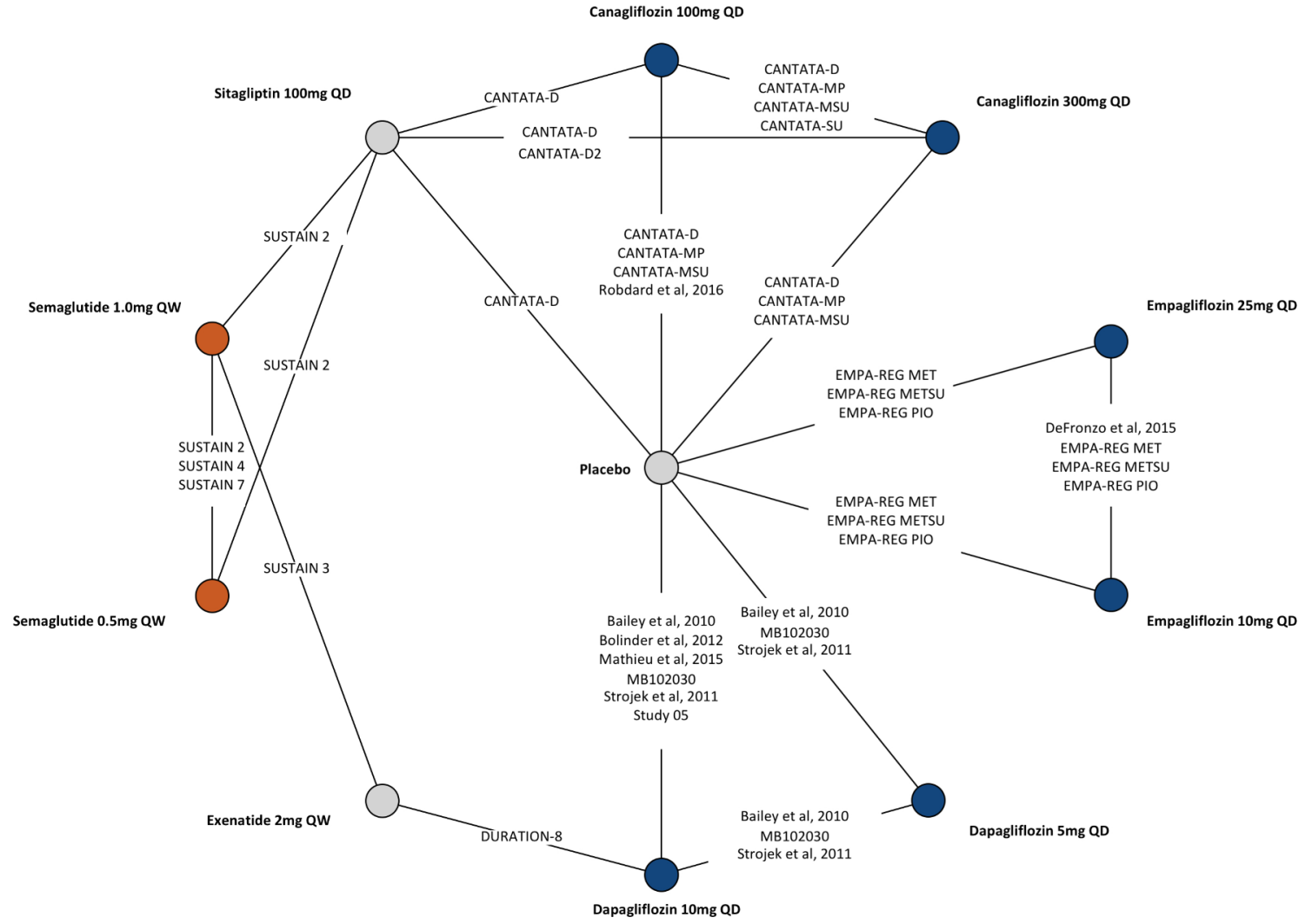

Figure 2 Overall network of evidence of semaglutide, SGLT-2is and other treatments for type II diabetes that is uncontrolled using 1-2 oral antidiabetics.

$\mathrm{HbA}_{1 \mathrm{c}}$ were also observed with once-weekly semaglutide $0.5 \mathrm{mg}$ compared with all high-dose and low-dose SGLT-2is of interest, but with smaller effect sizes than those observed with once-weekly $1.0 \mathrm{mg}$ dose. For all outcomes, modelled responses, rankings and the SUCRA scores are shown in the online supplementary appendix. Once-weekly semaglutide $1.0 \mathrm{mg}$ had the highest probability of being ranked first with a SUCRA score of 1 .

These results showed that while all therapies significantly reduced $\mathrm{HbA}_{1 \mathrm{c}} \mathrm{compared}$ with placebo, both once-weekly semaglutide doses demonstrated substantial clinical significance over the SGLT-2is comparators. A $0.3 \% \mathrm{HbA}_{1 \mathrm{c}}$ margin has been considered as threshold to assess clinical superiority between diabetes treatments by the FDA. ${ }^{46}$ Considering this threshold, our results show that once-weekly semaglutide $0.5 \mathrm{mg}$ had $\mathrm{HbA}_{1 \mathrm{c}}$ reductions over other SGLT-2is of between $0.30 \%$ and $0.70 \%$, and once-weekly semaglutide $1.0 \mathrm{mg}$ showed reductions between $0.56 \%$ and $0.95 \%$, suggesting that these results are clinically meaningful.

\section{Proportion of patients achieving $<7 \% \mathrm{HbA1c}$}

A total of 16 trials consisting of 8941 patients were included. ${ }^{19-21} 25 \quad 2633-36 \quad 38 \quad 3941-45$ The DIC difference between the fixed-effect and random-effect models was less than three. The fixed-effect model was preferred (DIC: 64.62) because the majority of the connections in this network were supported by one or two trials and a random-effect model is not feasible in such cases.
Similar to the results obtained in the change from baseline for $\mathrm{HbA}_{1 \mathrm{c}}$ analysis, once-weekly semaglutide was statistically significantly better than all SGLT-2is in achieving target $\mathrm{HbA}_{1 \mathrm{c}}$ levels of $<7 \%$ (table 4 ).

The OR for achieving target $\mathrm{HbA}_{1 c}<7 \%$ of once-weekly semaglutide $1.0 \mathrm{mg}$ versus SGLT-2is ranged from 2.80 for empagliflozin $25 \mathrm{mg}$ (95\% CrI: 1.74 to 4.52 ) to 5.52 for canagliflozin $100 \mathrm{mg}$ (95\% CrI: 3.91 to 7.81 ). Again, relative to the same treatments, the OR for target $\mathrm{HbA}_{1 \mathrm{c}}<7 \%$ of once-weekly semaglutide $0.5 \mathrm{mg}$ versus SGLT-2is ranged from 2.06 for empagliflozin $25 \mathrm{mg}$ (95\% CrI: 1.27 to 3.39 ) to 4.07 for canagliflozin $100 \mathrm{mg}$ (95\% CrI: 2.86 to 5.83). A SUCRA score of 1 was achieved for once-weekly semaglutide $1.0 \mathrm{mg}$ demonstrating that it was the best treatment compared with all other interventions.

\section{Change in weight $(\mathrm{kg})$}

Twenty trials comprising 10829 patients were included in the NMA for change from baseline in weight. $^{19-21}$ 25-28 30 33-39 41-45 The DIC difference between the fixed-effect and random-effect models was less than three. The fixed-effect model was chosen (DIC: 87.02) because the majority of the connections in this network were supported by one or two trials and a random-effect model is not feasible in such cases.

Once-weekly semaglutide displayed greater reductions in body weight compared with SGLT-2is. At $1.0 \mathrm{mg}$ dose, once-weekly semaglutide leads to statistically significant weight reduction relative to all SGLT-2is. Specifically, the 


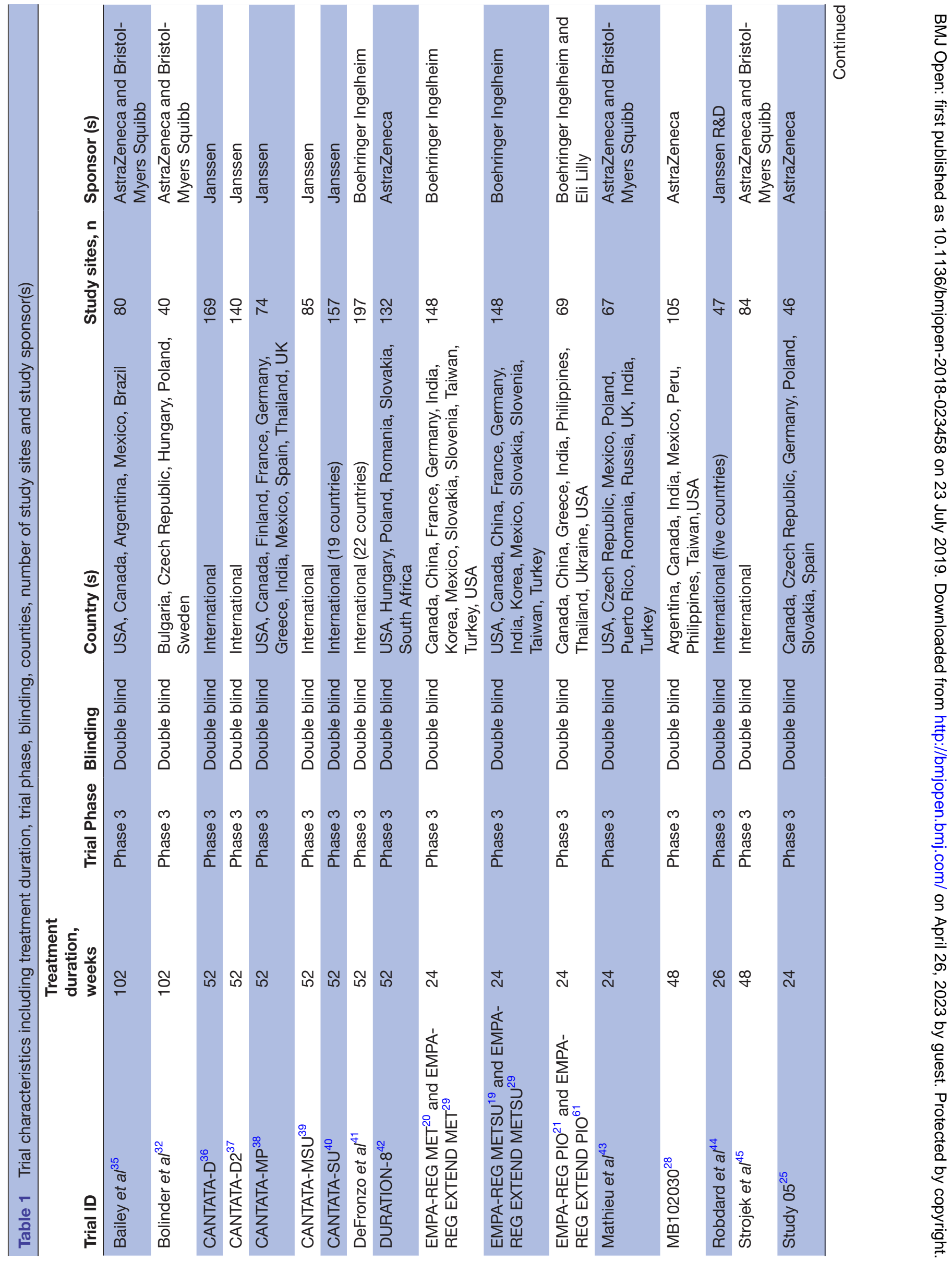




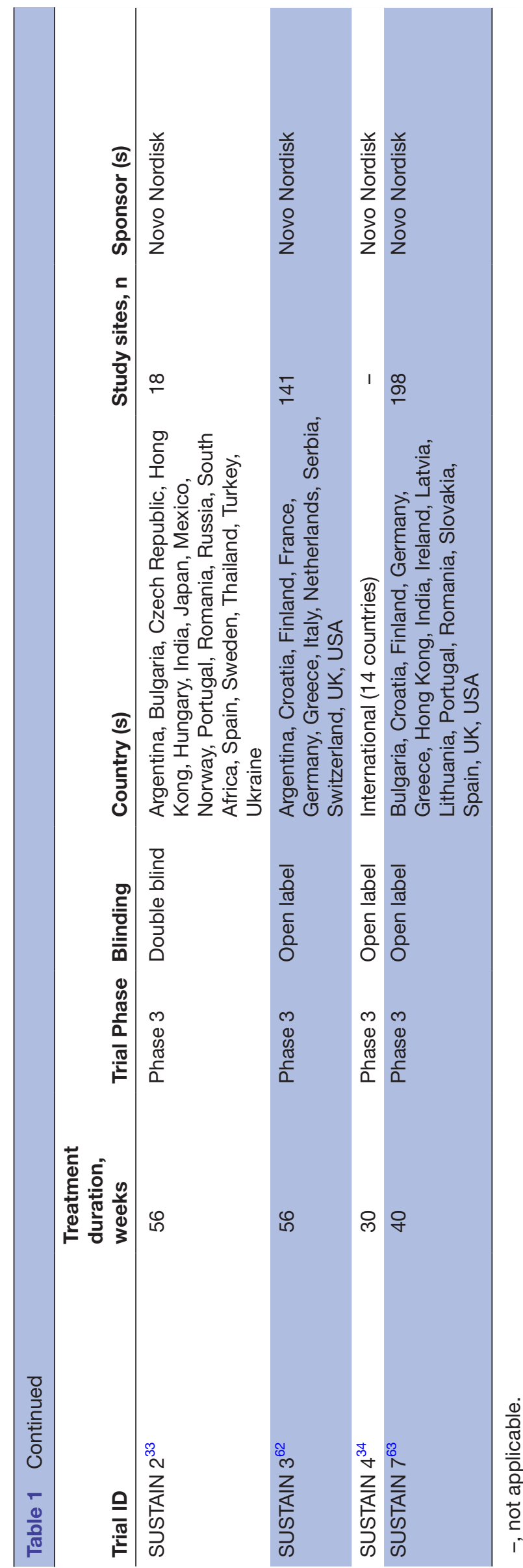

MD of once-weekly semaglutide $1.0 \mathrm{mg}$ versus SGLT-2is ranged from $-1.35 \mathrm{~kg}$ for canagliflozin $300 \mathrm{mg}(95 \% \mathrm{CrI}$ : -1.89 to $-0.79 \mathrm{~kg})$ to $-2.48 \mathrm{~kg}$ for dapagliflozin $5 \mathrm{mg}(95 \%$ CrI: -3.13 to $-1.82 \mathrm{~kg}$ ) (table 5 ).

With the $0.5 \mathrm{mg}$ dose of once-weekly semaglutide, the weight loss was comparable to the highest doses of canagliflozin $(300 \mathrm{mg}$ ) and empagliflozin $(25 \mathrm{mg})$, while the reductions were statistically significant in favour of once-weekly semaglutide $0.5 \mathrm{mg}$ when compared with canagliflozin $100 \mathrm{mg}$, empagliflozin $10 \mathrm{mg}$ and all doses of dapagliflozin (table 5). The results from the surface under the cumulative ranking curve demonstrated a SUCRA score of 1 for once-weekly semaglutide $1.0 \mathrm{mg}$ indicating that it was ranked as the best treatment with highest certainty.

\section{Change in SBP}

Seventeen trials including 9541 patients were included in NMA for change from baseline in SBP. ${ }^{19-21}$ 26-28 30 33-36 38 39 42-45 The DIC difference between the fixed-effect and random-effect models was less than three. The fixed-effect model was chosen (DIC: 71.12) for base-case analysis because the majority of the connections in this network were supported by one or two trials and a random-effect model is not feasible in such cases. Once-weekly semaglutide performed better than all SGLT-2is in reducing SBP, although with only the results of once-weekly semaglutide $1.0 \mathrm{mg}$ versus dapagliflozin $5 \mathrm{mg}$ (MD: $-3.12 \mathrm{~mm} \mathrm{Hg}$; 95\% CrI: -5.64 to $-0.60 \mathrm{~mm}$ $\mathrm{Hg}$ ) and empagliflozin $10 \mathrm{mg}$ (MD: $-2.45 \mathrm{~mm} \mathrm{Hg} ; 95 \%$ CrI: -4.75 to $-0.14 \mathrm{~mm} \mathrm{Hg}$ ) being statistically significant (table 5).

While exploring for between trial differences in patient characteristics, introducing covariate adjustments based on the number of prior $\mathrm{OAD}$ failures at baseline via metaregression yielded a better fitting model with lower DIC (67.92) compared with the base case (71.32). When adjusting for prior number of OADs received at baseline, the results were similar between once-weekly semaglutide and the SGLT-2i except for comparison of onceweekly semaglutide $1.0 \mathrm{mg}$ with respect to empagliflozin $10 \mathrm{mg}$ (MD: $-2.26 \mathrm{~mm} \mathrm{Hg}$; $95 \%$ CrI: -4.56 to $0.04 \mathrm{~mm}$ $\mathrm{Hg}$ ), where the results were not statistically significant anymore (see online supplementary appendix). Onceweekly semaglutide $1.0 \mathrm{mg}$ achieved a SUCRA score of 0.96 which suggests that it has a $96 \%$ probability of being ranked as the best treatment compared with all other interventions.

\section{Change in FPG}

Twenty-one trials including 11790 patients were included in the NMA for change from baseline in FPG. ${ }^{19-21}$ 25-28 $32-45$ The DIC difference between the fixed-effect and random-effect models was less than three. The fixed-effect model was chosen (DIC: 93.09) because the majority of the connections in this network were supported by one or two trials, and a random-effect model is not feasible in such cases. 


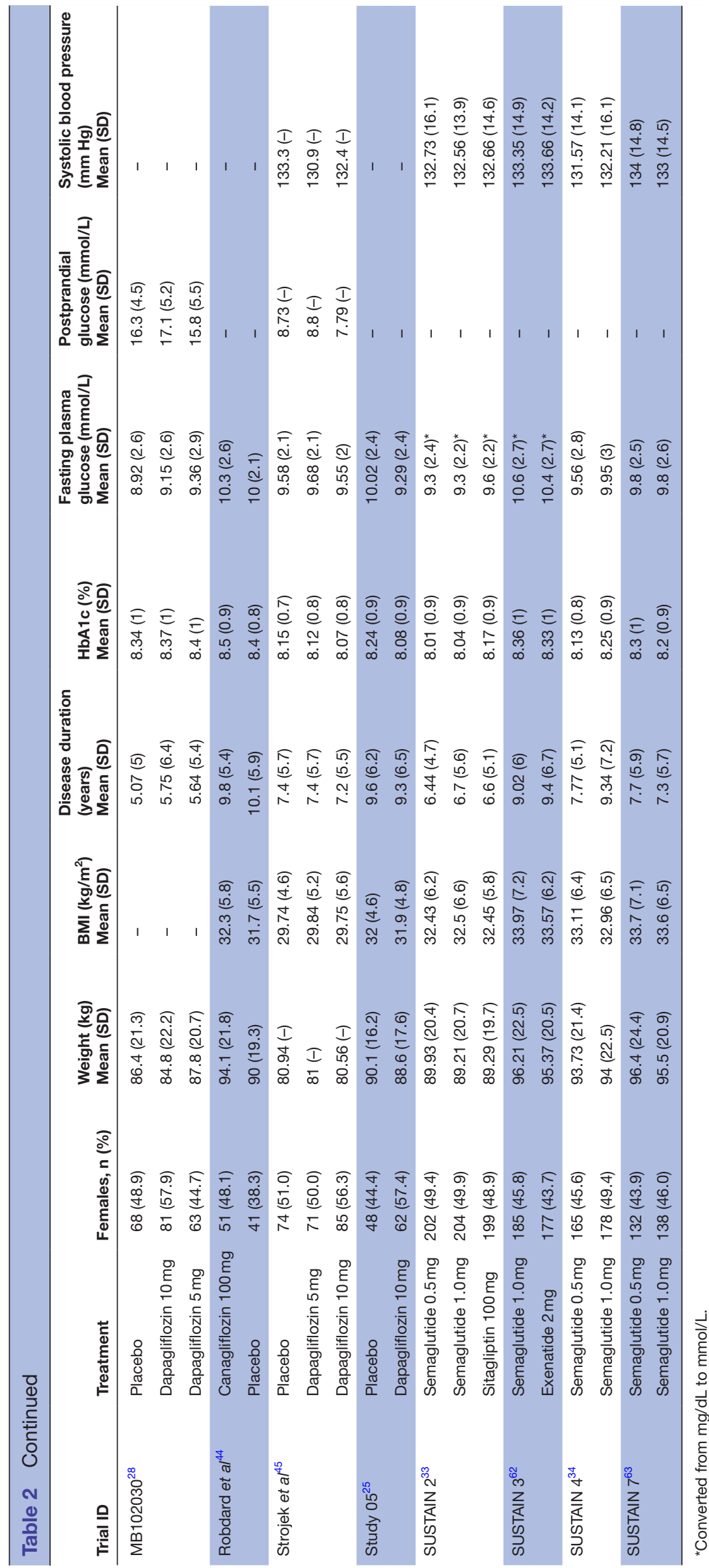


Table 3 Risk of bias assessment of included trials

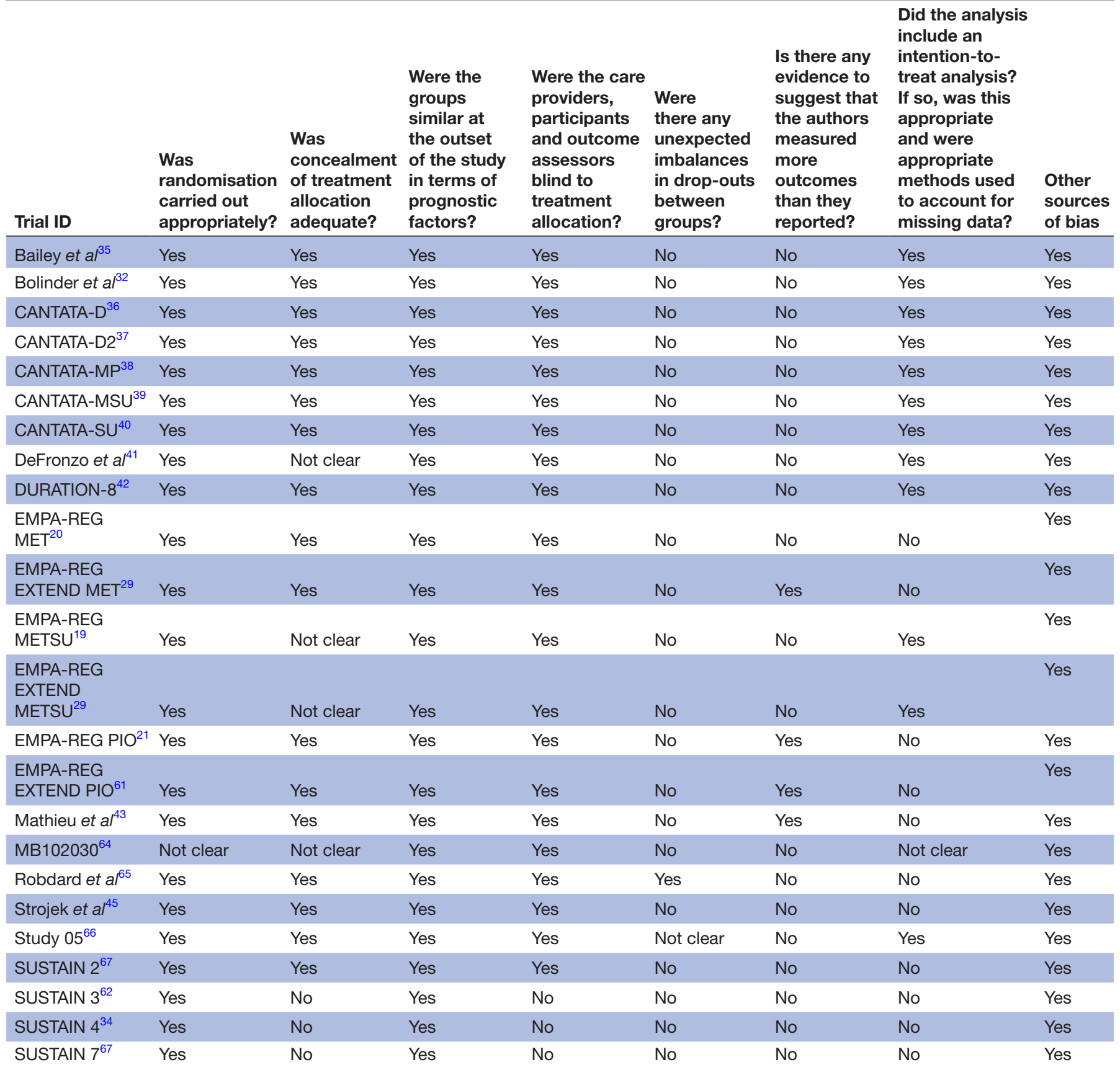

The high degree of 'Yes' status for the Other sources of bias are due to industry sponsoring. In modern pharmacological research, randomised controlled trials are typically industry sponsored. Nonetheless, there have been studies in the past that have indicated a tendency for higher effect sizes to be reported in such trials. As such, these have been identified as unclear with respect to risk of bias.

Once-weekly semaglutide performed better than all SGLT-2is; there was strong evidence of improved FPG relative to SGLT-2is for both doses of once-weekly semaglutide. Specifically, the MD of once-weekly semaglutide $1.0 \mathrm{mg}$ versus SGLT-2is ranged from $-0.41 \mathrm{mmol} / \mathrm{L}$ for canagliflozin $300 \mathrm{mg}$ ( $95 \%$ CrI: -0.72 to $-0.10 \mathrm{mmol} / \mathrm{L}$ ) to $-1.37 \mathrm{mmol} / \mathrm{L}$ for dapagliflozin $5 \mathrm{mg} \quad(95 \% \mathrm{CrI}$ : -1.76 to $-0.97 \mathrm{mmol} / \mathrm{L}$ ) (table 5 ). Once-weekly semaglutide $1.0 \mathrm{mg}$ achieved a full probability of being ranked as the best treatment as evident from the SUCRA score of 1 , which was consistent with most other efficacy outcomes.

\section{Sensitivity analyses and metaregressions}

Sensitivity analyses were conducted by: (i) removing three trials having a population composed of $>40 \%$ Asian patients ${ }^{19-21}$; and (ii) removing one trial with high cardiovascular risk. ${ }^{25}$ The results of these sensitivity analyses showed that whether or not Asian populations were 

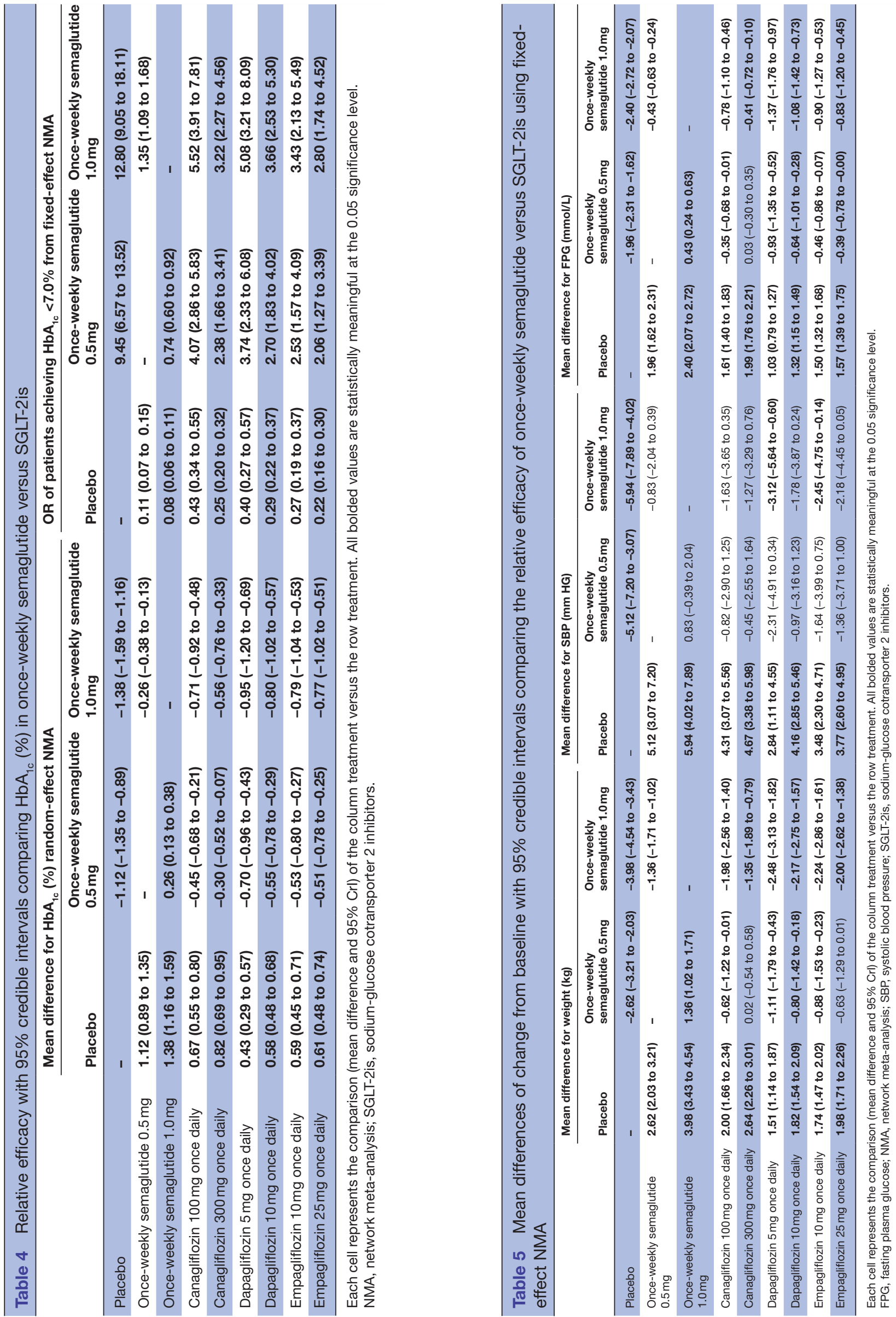

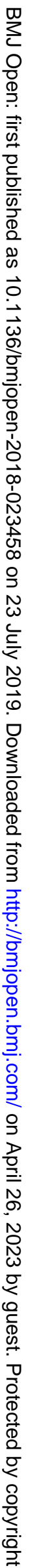


included, or if one trial with high cardiovascular risk was excluded, the effect estimates did not change in significance or direction for any of the outcomes of interest (online supplementary appendix). Similarly, metaregression did not lead to any meaningful change in estimate magnitudes or statistical significance, with the exception of SBP results (as shown above). Finally, safety analyses were not feasible as the safety results for SGLT-2is and semaglutide were reported at separate time points, namely 26 and 52 weeks. As such, safety analyses were not conducted.

\section{DISCUSSION}

Using an SLR and NMA, this study provided evidence in support of improved efficacy using once-weekly semaglutide relative to SGLT-2is licensed in Europe and North America for the treatment of patients with inadequate glycaemic control using 1-2 OADs. The SLR identified a rich evidence base via comprehensive literature searches. Results of the NMA demonstrated that across most efficacy outcomes $\left(\mathrm{HbA}_{1 c}\right.$, target $\mathrm{HbA1c}<7 \%$, weight loss and FPG), once-weekly semaglutide had the highest estimated efficacy. There was very strong evidence that once-weekly semaglutide led to larger decreases in both $\mathrm{HbA}_{1 \mathrm{c}}$ and weight. Specifically, the magnitude of the differences was large and clinically meaningful. With respect to SBP, SGLT-2is were also quite effective relative to placebo and though once-weekly semaglutide had the largest estimated beneficial effect, it could not quite be distinguished statistically from the SGLT-2is. There was also strong evidence of improved FPG relative to SGLT-2is for both doses of once-weekly semaglutide. Once-weekly semaglutide $0.5 \mathrm{mg}$ had the second best estimated relative efficacy for $\mathrm{HbA}_{1 \mathrm{c}}$ and weight.

Approximately $80 \%$ of patients with T2D are either overweight or obese, with associated issues for insulin resistance, glycaemic control and cardiovascular risk factors ${ }^{4}$; as a result, therapies which can lead to weight reduction or maintenance are desirable for the prevention of these complications. Although there is no universal standard as to a clinically relevant degree of weight reduction, weight loss above $2.3 \mathrm{~kg}$ is associated with improved comorbid outcomes. ${ }^{47} 48$ Every intervention included in the NMA was associated with weight reduction; however, only the results for the comparison of once-weekly semaglutide doses and canagliflozin $300 \mathrm{mg}$ to placebo were considered clinically meaningful. These results are consistent with the class-effects of GLP-1 receptor agonists compared with insulin or sulfonylureas. ${ }^{4}$ A study conducted by McIntosh et al evaluated second-line treatment after metformin observed an increase in weight for patients treated with sulfonylureas, meglitinides and thiazolidinediones compared with placebo and GLP-1 receptor agonists. ${ }^{49}$

All of the interventions had statistically significant reductions in SBP compared with placebo. A decrease of at least $5 \mathrm{~mm} \mathrm{Hg}$ in SBP is defined as clinically meaningful reduction ${ }^{50}$; compared with placebo, only once-weekly semaglutide and canagliflozin 300 and $100 \mathrm{mg}$ met this threshold. The results for SGLT-2is being associated with reductions in SBP are consistent with those from previously published meta-analyses. ${ }^{51-53}$

Choosing between treatment options requires a full consideration of the differences between treatments as well as patient preferences. This study has demonstrated that there are important advantages to once-weekly semaglutide relative to SGLT-2is with respect to clinical efficacy. These measures of efficacy include $\mathrm{Hb}_{\mathrm{Alc}}$, FPG and weight, which are both impactful on a number of known comorbidities, such as cardiovascular disease.

The strengths of this study include the use of highly sensitive searches in the peer-reviewed literature; this was supplemented with hand searches of leading diabetes research conferences. The review processes were determined by the predefined eligibility criteria established in the review protocol. Data quality was held at a high standard by the involvement of two independent researchers for the study selection and data extraction phases of the review; any discrepancies were managed by a third reviewer in the event of any disagreement. Additionally, a large number of sensitivity analyses (adjusted and restricted analyses) were conducted, demonstrating the robustness of the analytical results.

This study also has some limitations. The SLR is limited by the use of published data, leading to a risk of publication bias. Some clinical trials do not get published and others are only published in abstract form presenting limited information. The current study included an extensive search of conference abstracts to help mitigate the impact on the SLR results. Another limitation was the reliance on fixed-effect models, which may have underestimated the uncertainty around estimates. The choice of fixed-effect models were in accordance with NICE guidelines; however, for some outcomes network sparseness rather than lack of between study heterogeneity may have driven this choice. Therefore, results should be interpreted with care. Finally, with respect to the SLR, the search and selection process was restricted to trials published in the English language.

There were also several limitations to the analyses. First, while the population of interest was patients with inadequate glycaemic control using 1-2 OADs, a large number of studies only included patients on OAD monotherapy while others only included patients on dual therapy, which may have affected the homogeneity of the population. The pooling of such populations was required to ensure network connectivity, and models adjusting for these differences through metaregression suggest minimal impact from these differences. Second, there was a lack of direct comparisons due to a deficiency of reported comparisons in the literature. This placed a greater weight on the hypothesis of consistency between direct and indirect evidences. Nonetheless, despite longer pathways between the treatments of interest, the analysis still provided strong evidence of higher efficacy for 
once-weekly semaglutide and did not hint any evidence of inconsistency. Third, analyses of safety outcomes were not feasible; however, it should be noted that safety concerns are known to be different between these two drugs classes. SGLT-2is are known to have concerns over urinary and genital tract infections, volume depletion, ketoacidosis and thromboembolic events. ${ }^{545}$ Semaglutide has more concerns with gastrointestinal adverse events, hypoglycaemia, and amylase and lipase increases. ${ }^{56}$ Additionally, some studies have found that incretin-based drugs are more effective in Asian patients. ${ }^{57-59}$ Therefore, the results of the present NMA may not be extrapolated from European and North American populations directly to Asian populations. However, in the scope of the current study, sensitivity analyses around the proportion of Asian patients included in trials had negligible impact on results. Finally, these analyses only speak to the differences between these treatments with respect to clinical efficacy. Further research, including cost-effectiveness studies, may be informed by the current study estimates of relative efficacy, to assess impact on cost and quality of life. Whereas treatments may be compared on their relative efficacy using network meta-analysis, the long-term modelling of the outcomes for T2D and associated costs and health-related quality of life impact is needed in order to understand the benefits of antidiabetic drugs. ${ }^{55}$ Health economic evaluations of these treatments are currently underway, but for the sake of timeliness and interpretability, the current study focuses on clinical outcomes.

Once-weekly semaglutide demonstrated clinically meaningful reductions in $\mathrm{HbA}_{1 \mathrm{c}}$ and body weight in T2D patients inadequately controlled by 1-2 OADs, compared with all SGLT-2is licensed in Europe and North America, and was comparable to them in improving SBP. The evidence supports once-weekly semaglutide as a potential treatment option for patients inadequately controlled by 1-2 OADs. Given the lack of head-to-head evidence, this analysis provides valuable insight into the comparative outcomes of once-weekly semaglutide versus SGLT-2is. Nonetheless, these results do not preclude the need for more head-to-head studies investigating the comparative efficacy and safety of these therapies, as well as the benefits of their combination. ${ }^{3160}$

\section{Acknowledgements We would like to thank Ziyu Zhang and Robyn Kendall for} their contributions in the systematic literature review.

Contributors SK, EP, ED, LW, HV and SL participated in the conception and design of the work. SK, EP and ED participated in the collection of data. All authors participated in data analysis and interpretation. SK, RS, ED, LW, HV and SL were responsible for drafting the paper. All authors were involved in the critical revision of the paper, and all authors also gave final approval for this version of the paper to be published.

Funding This work was supported by Novo Nordisk A/S. No restrictions have been placed on the choice of analyses or the interpretation of the results.

Competing interests LW, HV and SL are employees of Novo Nordisk. RS, EP, ED and SK report funding provided by Novo Nordisk to Precision Xtract, where they are employees.

Patient consent for publication Not required.

Provenance and peer review Not commissioned; externally peer reviewed.
Data sharing statement All data used for these analyses are publicly available. Given the use of clinical Study Reports, data are not available. For further questions regarding data, please reach out to the corresponding author.

Open access This is an open access article distributed in accordance with the Creative Commons Attribution Non Commercial (CC BY-NC 4.0) license, which permits others to distribute, remix, adapt, build upon this work non-commercially, and license their derivative works on different terms, provided the original work is properly cited, appropriate credit is given, any changes made indicated, and the use is non-commercial. See: http://creativecommons.org/licenses/by-nc/4.0/.

\section{REFERENCES}

1. American Diabetes A; American Diabetes Association. Diagnosis and classification of diabetes mellitus. Diabetes Care 2012;35(Suppl 1):S64-S71.

2. Atlas ID, Federation ID. IDF). Brussels, Belgium: International Diabetes Federation 2017;2013.

3. Thrasher J. Pharmacologic Management of Type 2 Diabetes Mellitus: Available Therapies. Am J Cardiol 2017;120(1S):S4-S16.

4. Inzucchi SE, Bergenstal RM, Buse JB, et al. Management of hyperglycaemia in type 2 diabetes, 2015: a patient-centred approach. Update to a position statement of the American Diabetes Association and the European Association for the Study of Diabetes. Diabetologia 2015;58:429-42.

5. Excellence NIfHaC. Type 2 diabetes in adults: management. $\mathrm{NIfHaC}$ E, ed. United Kingdom, 2015.

6. National Institute for Health and Care Excellence in the UK. Type 2 diabetes in adults: management. NICE guideline [NG28] 2015 https:// www.nice.org.uk/guidance/ng28/resources (accessed 29 Dec 2016).

7. American Diabetes Association. Standards of Medical Care in Diabetes-2018 Abridged for Primary Care Providers. Clin Diabetes 2018;36:14-37.

8. Dias S, Sutton AJ, Ades AE, et al. Evidence synthesis for decision making 2: a generalized linear modeling framework for pairwise and network meta-analysis of randomized controlled trials. Med Decis Making 2013;33:607-17.

9. Jansen JP, Fleurence R, Devine B, et al. Interpreting indirect treatment comparisons and network meta-analysis for healthcare decision making: report of the ISPOR Task Force on Indirect Treatment Comparisons Good Research Practices: part 1. Value Health 2011;14:417-28.

10. Mills EJ, loannidis JP, Thorlund K, et al. How to use an article reporting a multiple treatment comparison meta-analysis. JAMA 2012;308:1246-53.

11. Lu G, Ades AE. Combination of direct and indirect evidence in mixed treatment comparisons. Stat Med 2004;23:3105-24.

12. Sharma R, Wilkinson L, Vrazic $H$, et al. Comparative efficacy of onceweekly semaglutide and SGLT-2 inhibitors in type 2 diabetic patients inadequately controlled with metformin monotherapy: a systematic literature review and network meta-analysis. Curr Med Res Opin 2018;34:1595-603.

13. Centre for Reviews and Dissemination. Systematic Reviews 2009 https://www.york.ac.uk/crd/guidance/ (accessed 29 Dec 2016).

14. Higgins, JPT. Cochrane Handbook for Systematic Reviews of Interventions Version 5.1.0. 2011 http://handbook.cochrane.org.

15. Nathan DM, Turgeon H, Regan S. Relationship between glycated haemoglobin levels and mean glucose levels over time. Diabetologia 2007;50:2239-44.

16. Jansen JP, Naci $\mathrm{H}$. Is network meta-analysis as valid as standard pairwise meta-analysis? It all depends on the distribution of effect modifiers. BMC Med 2013;11:159.

17. Spiegelhalter DJ, Best NG, Carlin BP, et al. Bayesian measures of model complexity and fit. Journal of the Royal Statistical Society: Series B 2002;64:583-639.

18. Dias S, Welton NJ, Caldwell DM, et al. Checking consistency in mixed treatment comparison meta-analysis. Stat Med 2010;29(78):932-44.

19. Häring HU, Merker L, Seewaldt-Becker E, et al. Empagliflozin as add-on to metformin plus sulfonylurea in patients with type 2 diabetes: a 24-week, randomized, double-blind, placebo-controlled trial. Diabetes Care 2013;36:3396-404.

20. Häring HU, Merker L, Seewaldt-Becker E, et al. Empagliflozin as add-on to metformin in patients with type 2 diabetes: a 24-week, randomized, double-blind, placebo-controlled trial. Diabetes Care 2014;37:1650-9.

21. Kovacs CS, Seshiah V, Swallow R, et al. Empagliflozin improves glycaemic and weight control as add-on therapy to pioglitazone or pioglitazone plus metformin in patients with type 2 diabetes: a 24- 
week, randomized, placebo-controlled trial. Diabetes Obes Metab 2014;16:147-58

22. Chan JC, Malik V, Jia W, et al. Diabetes in Asia: epidemiology, risk factors, and pathophysiology. JAMA 2009;301:2129-40.

23. Malik VS, Willett WC, Hu FB. Global obesity: trends, risk factors and policy implications. Nat Rev Endocrinol 2013;9:13-27.

24. Chen KW, Boyko EJ, Bergstrom RW, et al. Earlier appearance of impaired insulin secretion than of visceral adiposity in the pathogenesis of NIDDM. 5-Year follow-up of initially nondiabetic Japanese-American men. Diabetes Care 1995;18:747-53.

25. Matthaei S, Bowering K, Rohwedder K, et al. Dapagliflozin improves glycemic control and reduces body weight as add-on therapy to metformin plus sulfonylurea: a 24-week randomized, double-blind clinical trial. Diabetes Care 2015;38:365-72.

26. Ahmann AJ, Capehorn M, Charpentier G, et al. Efficacy and Safety of Once-Weekly Semaglutide Versus Exenatide ER in Subjects With Type 2 Diabetes (SUSTAIN 3): A 56-Week, Open-Label, Randomized Clinical Trial. Diabetes Care 2018;41:258-66.

27. Pratley RE, Aroda VR, Lingvay I, et al. Semaglutide versus dulaglutide once weekly in patients with type 2 diabetes (SUSTAIN 7): a randomised, open-label, phase $3 \mathrm{~b}$ trial. Lancet Diabetes Endocrinol 2018;6:275-86.

28. Rosenstock J, Vico M, Wei L, et al. Effects of dapagliflozin, an SGLT2 inhibitor, on $\mathrm{HbA}(1 \mathrm{c})$, body weight, and hypoglycemia risk in patients with type 2 diabetes inadequately controlled on pioglitazone monotherapy. Diabetes Care 2012;35:1473-8.

29. Haering HU, Merker L, Christiansen AV, et al. Empagliflozin as addon to metformin plus sulphonylurea in patients with type 2 diabetes. Diabetes Res Clin Pract 2015;110:82-90.

30. Bolinder J, Ljunggren Ö, Johansson L, et al. Dapagliflozin maintains glycaemic control while reducing weight and body fat mass over 2 years in patients with type 2 diabetes mellitus inadequately controlled on metformin. Diabetes Obes Metab 2014;16:159-69.

31. Frías JP, Guja C, Hardy E, et al. Exenatide once weekly plus dapagliflozin once daily versus exenatide or dapagliflozin alone in patients with type 2 diabetes inadequately controlled with metformin monotherapy (DURATION-8): a 28 week, multicentre, double-blind, phase 3, randomised controlled trial. Lancet Diabetes Endocrinol 2016;4:1004-16.

32. Bolinder J, Ljunggren Ö, Kullberg J, et al. Effects of dapagliflozin on body weight, total fat mass, and regional adipose tissue distribution in patients with type 2 diabetes mellitus with inadequate glycemic control on metformin. J Clin Endocrinol Metab 2012;97:1020-31.

33. Ahren B, Comas L, Kumar H, et al, 2016. Efficacy and safety of onceweekly semaglutide versus sitagliptin as add-on to metformin and/ or thiazolidinediones after 56 weeks in subjects with type 2 diabetes (SUSTAIN 2). 76th American Diabetes Association conference. New Orleans, LA

34. DeVries J, Bain S, Cariou B, et al, 2016. Efficacy and safety of onceweekly semaglutide versus once-daily insulin glargine in insulin-naïve subjects with type 2 diabetes (SUSTAIN 4). 52nd EASD conference. Munish Germany

35. Bailey CJ, Gross JL, Pieters A, et al. Effect of dapagliflozin in patients with type 2 diabetes who have inadequate glycaemic control with metformin: a randomised, double-blind, placebo-controlled trial. Lancet 2010;375:2223-33.

36. Lavalle-González FJ, Januszewicz A, Davidson J, et al. Efficacy and safety of canagliflozin compared with placebo and sitagliptin in patients with type 2 diabetes on background metformin monotherapy: a randomised trial. Diabetologia 2013;56:2582-92.

37. Schernthaner G, Gross JL, Rosenstock J, et al. Canagliflozin compared with sitagliptin for patients with type 2 diabetes who do not have adequate glycemic control with metformin plus sulfonylurea: a 52-week randomized trial. Diabetes Care 2013;36:2508-15.

38. Forst T, Guthrie R, Goldenberg R, et al. Efficacy and safety of canagliflozin over 52 weeks in patients with type 2 diabetes on background metformin and pioglitazone. Diabetes Obes Metab 2014;16:467-77.

39. Wilding JP, Charpentier G, Hollander P, et al. Efficacy and safety of canagliflozin in patients with type 2 diabetes mellitus inadequately controlled with metformin and sulphonylurea: a randomised trial. Int $J$ Clin Pract 2013;67:1267-82.

40. Cefalu WT, Leiter LA, Yoon KH, et al. Efficacy and safety of canagliflozin versus glimepiride in patients with type 2 diabetes inadequately controlled with metformin (CANTATA-SU): 52 week results from a randomised, double-blind, phase 3 non-inferiority trial. Lancet 2013;382:941-50.

41. DeFronzo RA, Lewin A, Patel S, et al. Combination of empagliflozin and linagliptin as second-line therapy in subjects with type 2 diabetes inadequately controlled on metformin. Diabetes Care 2015;38:384-93.
42. Guja C, Frías JP, Ahmed A, et al. DURATION-8 Randomized Controlled Trial 1-Year Results: Efficacy and Safety of Once-Weekly Exenatide (ExQW) Plus Once-Daily Dapagliflozin (DAPA) Versus ExQW or DAPA alone. 2017.

43. Mathieu C, Ranetti AE, Li D, et al. Randomized, Double-Blind, Phase 3 Trial of Triple Therapy With Dapagliflozin Add-on to Saxagliptin Plus Metformin in Type 2 Diabetes. Diabetes Care 2015;38:2009-17.

44. Rodbard HW, Seufert J, Aggarwal N, et al. Efficacy and safety of titrated canagliflozin in patients with type 2 diabetes mellitus inadequately controlled on metformin and sitagliptin. Diabetes Obes Metab 2016;18:812-9.

45. Strojek K, Yoon $\mathrm{KH}$, Hruba V, et al. Effect of dapagliflozin in patients with type 2 diabetes who have inadequate glycaemic control with glimepiride: a randomized, 24-week, double-blind, placebocontrolled trial. Diabetes Obes Metab 2011;13:928-38.

46. U.S. Food and Drug Administration. Guidance for Industry, Diabetes Mellitus: developing drugs and therapeutic biologics for treatment and prevention. In: Services UDoHaH, ed 2008.

47. Sherwood NE, Jeffery RW, French SA, et al. Predictors of weight gain in the Pound of Prevention study. Int J Obes Relat Metab Disord 2000;24:395-403.

48. Unick JL, Beavers D, Jakicic JM, et al. Effectiveness of lifestyle interventions for individuals with severe obesity and type 2 diabetes: results from the Look AHEAD trial. Diabetes Care 2011;34:2152-7.

49. Mclntosh B, Cameron C, Singh SR, et al. Second-line therapy in patients with type 2 diabetes inadequately controlled with metformin monotherapy: a systematic review and mixed-treatment comparison meta-analysis. Open Med 2011;5:e35-48.

50. Reboldi G, Gentile G, Angeli F, et al. Effects of intensive blood pressure reduction on myocardial infarction and stroke in diabetes: a meta-analysis in 73,913 patients. J Hypertens 2011;29:1253-69.

51. Baker WL, Smyth LR, Riche DM, et al. Effects of sodium-glucose cotransporter 2 inhibitors on blood pressure: a systematic review and meta-analysis. J Am Soc Hypertens 2014;8(4):262-75.

52. Mearns ES, Saulsberry WJ, White CM, et al. Efficacy and safety of antihyperglycaemic drug regimens added to metformin and sulphonylurea therapy in Type 2 diabetes: a network meta-analysis. Diabet Med 2015;32:1530-40.

53. Turnbull F; Blood Pressure Lowering Treatment Trialists' Collaboration. Effects of different blood-pressure-lowering regimens on major cardiovascular events: results of prospectively-designed overviews of randomised trials. Lancet 2003;362:1527-35.

54. Hsia DS, Grove O, Cefalu WT. An update on sodium-glucose cotransporter-2 inhibitors for the treatment of diabetes mellitus. Curr Opin Endocrinol Diabetes Obes 2017;24:73-9.

55. Yabe D, Kuwata H, Usui R, et al. Glucagon-like peptide-1 receptor agonist therapeutics for total diabetes management: assessment of composite end-points. Curr Med Res Opin 2015;31:1267-70.

56. Nordisk N. Ozempic - semaglutide injection [Product insert]. Copenhagen Denmark: Novo Nordisk, 2018.

57. Kim YG, Hahn S, Oh TJ, et al. Differences in the glucose-lowering efficacy of dipeptidyl peptidase-4 inhibitors between Asians and non-Asians: a systematic review and meta-analysis. Diabetologia 2013;56:696-708.

58. Kim YG, Hahn S, Oh TJ, et al. Differences in the HbA1c-lowering efficacy of glucagon-like peptide-1 analogues between Asians and non-Asians: a systematic review and meta-analysis. Diabetes Obes Metab 2014:16:900-9.

59. Seino Y, Kuwata H, Yabe D. Incretin-based drugs for type 2 diabetes: Focus on East Asian perspectives. J Diabetes Investig 2016;7(Suppl 1):102-9.

60. Seino Y, Yabe D, Sasaki T, et al. Sodium-glucose cotransporter-2 inhibitor luseogliflozin added to glucagon-like peptide 1 receptor agonist liraglutide improves glycemic control with bodyweight and fat mass reductions in Japanese patients with type 2 diabetes: A 52-week, open-label, single-arm study. J Diabetes Investig 2018:9:332-40.

61. Kovacs CS, Seshiah V, Merker L, et al. Empagliflozin as Add-on Therapy to Pioglitazone With or Without Metformin in Patients With Type 2 Diabetes Mellitus. Clin Ther 2015;37:1773-88.

62. Ahmann A, Capehorn M, Charpentier G, et al, 2016. Efficacy and safety of once-weekly semaglutide versus exenatide ER after 56 weeks in subjects with type 2 diabetes (SUSTAIN 3). 76th American Diabetes Association conference. New Orleans, LA

63. Pratley R, Viljoen A. Efficacy and safety of semaglutide versus dulaglutide as add-on to metformin in subjects with type 2 diabetes. 2017.

64. Rosenstock J, Hansen L, Zee P, et al. Dual add-on therapy in type 2 diabetes poorly controlled with metformin monotherapy: a randomized double-blind trial of saxagliptin plus dapagliflozin 
addition versus single addition of saxagliptin or dapagliflozin to metformin. Diabetes Care 2015;38:376-83.

65. Rodbard W, Lingvay R, Reed J, et al, 2016. Efficacy and safety of semaglutide once-weekly versus placebo as add-on to basal insulin alone or in combination with metformin in subjects with type 2 diabetes (SUSTAIN 5). 52nd EASD Conference. Munich, Germany

66. Grandy S, Sternhufvud C, Ryden A, et al. Patient-reported outcomes among patients with type 2 diabetes mellitus treated with dapagliflozin in a triple-therapy regimen for 52 weeks. Diabetes Obes Metab 2016;18:306-9.

67. Ahrén $\mathrm{B}$, Masmiquel L, Kumar $\mathrm{H}$, et al. Efficacy and safety of once-weekly semaglutide versus once-daily sitagliptin as an add-on to metformin, thiazolidinediones, or both, in patients with type 2 diabetes (SUSTAIN 2): a 56-week, double-blind, phase 3a, randomised trial. Lancet Diabetes Endocrinol 2017;5:341-54. 